Ann. Sci. forest., 1981, 38 (3), 355-375

\title{
Déterminisme génétique de la croissance en hauteur du Pin maritime (Pinus pinaster Ait) \\ III. - Evolution des composantes de la variance phénotypique et génotypique
}

\author{
A. KREMER \\ I.N.R.A., Laboratoire d'Amélioration des Arbres forestiers, \\ Pierroton, F 33610 Cestas
}

\begin{abstract}
Résumé
Cette étude se propose de suivre l'évolution des composantes de la variance phénotypique et génotypique de l'accroissement en hauteur en utilisant le coefficient de variation exprimé au niveau de chaque composante, l'accroissement en hauteur étant estimé par le cumul des accroissements successifs. Le coefficient de variation de l'erreur, de l'effet station, de l'interaction famille $\times$ station et de l'interaction mère $\times$ père décroît avec le temps alors que lit variance des effets principaux reste stable. Jusquà 8 ans, cette évolution semble due à l'effet d'âge et s'explique par le changement des conditions du milieu au cours du temps. Les conséquences au niveau des paramètres génétiques sont immédiates : augmentation de l'héritabilité avec le cumul et diminution du rapport $\mathrm{V}_{\mathrm{D}} / \mathrm{V}_{\mathrm{G}}$ à tel point que tout accroissement du cumul d'ordre $n$ est meilleur prédicteur d'un accroissement de cumul d'ordre inférieur à n que ce dernier lui-même. Par ailleurs, au niveau de la décomposition de la variance génotypique, la variance d'additivité est plus importante que la variance de dominance. Enfin, la possibilité de prévoir l'aptitude spécifique à la combinaison (ASC) à partir de l'aptitude générale à la combinaison ( $\mathrm{AGC}$ ) des deux parents est envisagée.
\end{abstract}

$$
\text { Mots-clés }
$$

Modèle génétique, variance phénotypique, génotypique, d'additivité ét de dominance, aptitude générale à la combinaison, aptitude spécifique à la combinaison, héritabilitś.

Coefficient de prédiction génétique

\section{Introduction}

L'efficacité de la sélection dépend en grande partie de la maîtrise que le sélectionneur peut exercer sur les composantes de la variabilité phénotypique du matériel végétal qu'il gère. L'un des objectifs de cette maîtrise est de diminuer la part du milieu dans l'expression du caractère en cause et ceci généralement par la sophistication des dispositifs expérimentaux.

Chez les plantes pérennes la réduction de la variation due au milieu peut être optimisée par la recherche de la «situation biologique» au cours de laquelle le 
milieu affecterait dans une moindre mesure lexpression du caractère et où ce dernier serait le meilleur prédicteur du caractère adulte. Le même raisonnement peut bien évidemment être fait au niveau de la variabilité génotypique que l'on souhaiterait maximiser. Et, à ce stade, l'intérêt peut être porté sur la composante de la variabilité génotypique (additive ou non additive) que le schéma d'amélioration veut exploiter. La présente contribution se propose d'étudier l'évolution des composantes de la variance phénotypique et génotypique pour la croissance en hauteur du pin maritime. $\mathrm{Ce}$ caractère, mesuré par le cumul d’un nombre maximum de pousses au-delà de la cinquième année de végétation est utilisé comme critère de sélection, meilleur prédicteur de la croissance en volume.

De nombreux résultats issus de tests de descendances avaient montré que jusqu`à cet âge, l'effet génotypique n'expliquait qu'une partie infime de la variance phénotypique. Ces observations étaient généralement attribuées soit à des effets maternels (grosseur de graines) soit à des modalités de croissance particulières (croissance fixe ou libre selon que les entre-nœuds sont préformés ou non). Une formulation plus générale désignait ce comportement par le terme «croissance juvénile».

Deux précédentes études (Kremer, 1981 a et b) avaient souligné l’importance du polycyclisme sur la croissance globale et sur la régularité interannuelle des accroissements successifs : ces études de caractère comportemental ou plus biologique laissaient clairement apparaître la nécessité de cumuler les accroissements sur un nombre important d'années pour avoir une meilleure estimation de la croissance en hauteur. En suivant l'évolution des composantes au cours des cumuls successifs à partir de la cinquième année de végétation, cette étude se fixe donc pour objectif de :

- aborder l'aspect génétique de sélection;

- approcher la situation optimale pour la sélection :

- comparer l'évolution des composantes de la croissance phénotypique avec l'évolution des conditions environnementales et des modalités de croissance du pin maritime ;

- déterminer la part de la variation génotypique utilisable en sélection et comment l'utiliser.

\section{1. - Matériel}

Les trois dispositifs expérimentaux utilisés pour cette étude ont déjà été décrits dans une précédente publication (Kremer, 1981 a). En rappel voici les éléments principaux de ces dispositifs :

2.44.3.1 : 100 familles de demi-frères installées en lande humide (Saint-Alban) selon un triple lattice $10 \times 10$ répété 3 fois. Mesures d'accroissement en hauteur successifs entre la $7^{\circ}$ et la $12^{\circ}$ année de végétation (1971 à 1976) réalisées sur un échantillon systématique de 3 plants par parcelle unitaire (P.U.).

2.44.3.2. : 100 familles de demi-frères, toutes communes au 2.44.3.1. installées en dune (Soustons) selon un triple lattice $10 \times 10$ répété 2 fois. Mesures d'accroissement en hauteur successifs entre la $9^{\prime \prime}$ et la $12^{\prime \prime}$ année de végétation (1973 à 1976), réalisées sur un échantillon systématique de 4 plants par P.U. 
2.44.10. : 169 familles dont 155 appartenant à deux plans de croisement factoriel (K. Hinkelmann \& K. Stern, 1960: 4 pères et 21 mères chacun). Le dispositif est un lattice équilibré $13 \times 13$ répété 7 fois. Mesures d'accroissements en hauteur successifs entre la $5^{\circ}$ et la $8^{\circ}$ année de végétation (1973 à 1976) réalisées sur un échantillon systématique de 4 plants par P.U. Dans le premier plan trois familles ne sont pas représentées, dans le second ce nombre est de 10.

Lévolution de la croissance en hauteur est estimée par le cumul des accroissements annuels successifs. Dans le texte, on appellera l'accroissement de cumul d'ordre 1, l'accroissement d'une année donnée et l'accroissement du cumul d’ordre n, les accroissements cumulés sur $n$ années successives.

\section{2. - Méthodes}

Les données ont été traitées en deux étapes :

- Le programme ENVIR permet l'ajustement des données primaires à l'effet macrobloc et s’applique à tous les dispositifs déséquilibrés ou non, orthogonaux ou non.

Dans le cas présent les dispositifs ont été interprétés en blocs complets ( 9 pour le 2.44.3.1., 6 pour le 2.44.3.2. et 7 pour le 2.44.10), cas particulier traité par ENVIR.

$Y_{i j k}=\imath+F_{i}+b_{j}+(F b)_{i j}+E_{i j k}$

$F_{i}=$ effet famille (aléatoire)

$b_{i}=$ effet bloc (fixe)

$(\mathrm{Fb})_{\mathrm{ij}}=$ effet interaction famille $\times$ bloc

$\mathbf{E}_{\mathrm{ijk}}=$ erreur

Dans le cas présent:

$\mathrm{Y}_{\mathrm{ijk}}$ ajusté $=\mathrm{X}_{\mathrm{ijk}}=\mathrm{Y}_{\mathrm{ijk}}-\mathrm{b}_{\mathrm{i}}$

- La décomposition de la variance phénotypique et de la variance génotypique au niveau de chaque dispositif.

L'utilisation du modèle génétique telle qu'elle est faite ici suppose acquises un certain nombre d'hypothèses :

- il y a équilibre de linkage;

- il n'y a pas d'épistasie ;

- il n'y a pas d'effets maternels ni d'effet d'environnement commun aux familles :

- les parents des différents dispositifs ont été choisis au hasard dans une population en équilibre panmictique;

- les descendants ne sont pas consanguins.

Aucun élément ne permet de répondre aux trois premières hypothèses. En ce qui concerne la quatrième, les parents ont été sélectionnés phénotypiquement en forêt pour la hauteur et donc pour la croissance. Mais cette sélection en forêt s'est avérée inefficace. On peut donc considérer la population parentale comme un échantillon aléatoire de la population landaise. 
En ce qui concerne la quatrième hypothèse, il $\mathrm{y}$ a effectivement un risque de trouver des individus consanguins dans les familles de demi-frères (2.44.3.1. et 2.44.3.2.) issues de pollinisation libre en forêt. L'utilisation de gènes marqueurs a montré cependant que le taux d'autofécondation naturelle chez le Pin maritime était faible (BARADAT, comm. pers.). Si les hypothèses paraissent très restrictives, la valeur prédictive du modèle n'est pas pour autant remise en cause. Et c'est ce dernier aspect qui en définitive est le plus utile en sélection.

2.44.3.1. ou 2.44.3.2. (programme PINEDE)

$$
\begin{aligned}
& X_{i j}=\ell+F_{i}+E_{i j} \\
& \hat{V}_{\mathbf{P h}}=\hat{V}_{\mathbf{F}}+\dot{V}_{\mathbf{E}} \\
& F_{i}=\text { effet famille } N\left(0, V_{\mathbf{F}}\right) \\
& E_{i j}=\text { erreur } N\left(0, V_{E}\right) \\
& V_{\mathbf{P}^{\prime} \mathbf{h}}=\text { variance phénotypique }
\end{aligned}
$$

Les covariances entre apparentés s'écrivent :

$\operatorname{Cov}\left(X_{i j}, X_{i j}\right)=V_{F}=\frac{1}{4} V_{\Lambda} \quad V_{\Lambda}=$ variance additive

d'où une estimation de l'héritabilité :

$\hat{\mathrm{h}}^{2}=4 \frac{\hat{\mathrm{V}}_{\mathrm{F}}}{\hat{\mathrm{V}}_{\mathrm{F}}+\hat{\mathrm{V}}_{\mathrm{l}}}$

2.44.3.1. et 2.44.3.2. (programme CASOAR, option interaction génotype $\times$ milieu)

Effet station fixe

$$
\begin{aligned}
& X_{i j k}=\mu+F_{i}+s_{j}+(F s)_{j j}+E_{i j k} \\
& \hat{V}_{l^{\prime} h}=\hat{V}_{F^{\prime}}+\hat{V}_{\mathrm{ls}}+\hat{V}_{E} \\
& \hat{h}^{2}=4 \frac{\hat{V}_{F^{\prime}}}{\hat{V}_{\mathrm{r}}+\hat{V}_{\mathrm{rs}}+\hat{V}_{\mathrm{E}}}
\end{aligned}
$$

Effet station aléatoire

$$
\begin{aligned}
& X_{i j k}=\mu+F_{i}+S_{j}+(F S)_{i j}+E_{i j k} \\
& \hat{V}_{\mathrm{P}^{\prime} \mathbf{l}}=\hat{V}_{\mathrm{F}}+\hat{\mathrm{V}}_{\mathrm{S}}+\hat{\mathrm{V}}_{\mathrm{Fs}}+\hat{\mathrm{V}}_{\mathrm{E}} \\
& \hat{\mathrm{h}}^{2}=4 \frac{\hat{\mathrm{V}}_{\mathrm{F}}}{\hat{\mathrm{V}}_{\mathrm{F}}+\hat{\mathrm{V}}_{\mathrm{s}}+\hat{\mathrm{V}}_{\mathrm{F} \mathrm{s}}+\hat{\mathrm{V}}_{\mathrm{E}}}
\end{aligned}
$$


2.44.10. (programme CASOAR option plan de croisement :

analyse de variance multivariable non orthogonale [méthode HENDERSON III])

$$
\begin{aligned}
& \mathbf{X}_{\mathrm{ijk}}=\mu+\mathrm{M}_{\mathrm{i}}+\mathrm{P}_{\mathrm{j}}+(\mathrm{MP})_{\mathrm{ij}}+\mathrm{E}_{\mathrm{ijk}} \\
& \hat{\mathrm{V}}_{\mathrm{P}^{\prime} \mathrm{l}}=\hat{\mathrm{V}}_{\mathrm{M}}+\hat{\mathrm{V}}_{\mathrm{P}}+\hat{\mathrm{V}}_{\mathrm{MP}}+\hat{\mathrm{V}}_{\mathrm{E}} \\
& \mathrm{M}_{\mathrm{i}}=\text { effet mère } \mathrm{N}\left(0, \mathrm{~V}_{\mathrm{M}}\right) \quad \mathrm{i}: \mathbf{1} \ldots \mathrm{M} \\
& P_{j}=\text { effet père } N\left(0, V_{1}\right) \quad j: 1 \ldots P \\
& (\mathrm{MP})_{\mathrm{ij}}=\text { effet interaction mère } \times \text { père } \mathrm{N}\left(0, \mathrm{~V}_{\mathrm{MP}}\right) \\
& \mathrm{E}_{\mathrm{ijk}}=\text { erreur } \mathrm{N}\left(0, \mathrm{~V}_{\mathrm{F}}\right)
\end{aligned}
$$

Dans un plan de croisement factoriel, les différentes covariances entre apparentés s'écrivent de la manière suivante et conduisent à 2 estimations de $V_{A}$ (descendants de même mère et descendants de même père).

$\operatorname{Cov}\left(X_{i, k}, x_{i j^{\prime} k^{\prime}}\right)=V_{M}=\frac{1}{4} V_{A} \quad\left(V_{A}:\right.$ variance additive $)$

$\operatorname{Cov}\left(X_{i, j k}, X_{i^{\prime}, j k^{\prime}}\right)=V_{l^{\prime}}=\frac{1}{4} V_{A}$

$\operatorname{Cov}\left(X_{i, j}, X_{i, j k^{\prime}}\right)-\operatorname{Cov}\left(X_{i, j k}, X_{i, j^{\prime} i^{\prime}}\right)-\operatorname{Cov}\left(X_{i j l i}, X_{i^{\prime} j^{\prime}}\right)=V_{M r^{\prime}}=\frac{1}{4} V_{1}$

$\left(\mathrm{V}_{\mathrm{D}}\right.$ : variance de dominance)

d'où les estimations de $V_{A}$ et de $V_{1}$, et $V_{G}$ (variance génotypique)

$$
\begin{aligned}
& \hat{\mathrm{V}}_{\mathrm{A}}=4 \frac{(\mathrm{M}-1) \hat{\mathrm{V}}_{\mathrm{M}}+(\mathrm{P}-1) \hat{\mathrm{V}}_{\mathrm{P}}}{(\mathrm{M}-1)+(\mathrm{P}-1)} \\
& \hat{\mathrm{V}}_{\mathrm{D}}=4 \hat{\mathrm{V}}_{\mathrm{MI}} \\
& \hat{\mathrm{V}}_{\mathrm{G}}=\hat{\mathrm{V}}_{\mathrm{A}}+\hat{\mathrm{V}}_{\mathrm{D}}
\end{aligned}
$$

Les estimations de l'héritabilité $\left(\mathrm{V}_{\Lambda} / \mathrm{V}_{\mathrm{P} 11}\right)$ et de l'importance relative de la variance de dominance dans la variance génotypique sont alors immédiates.

\section{3. - Résultats}

\subsection{Décomposition de la variance phénotypique}

L'évolution des composantes de la variabilité phénotypique au cours du temps peut être appréhendée par l'estimation des valeurs successives des coefficients 
de variation exprimée aux différents niveaux (erreur, inter-famille, etc.). Ces valeurs sont représentées dans les figures $1_{A}, \mathbf{B}, \mathrm{C}$

$$
\mathrm{CV}_{\mathrm{F}}=\frac{\sqrt{ } \mathrm{V}_{\mathrm{F}}^{-}}{\overline{\mathrm{X}}} \quad \mathrm{CV}_{\mathrm{E}}=\frac{\sqrt{ } \mathrm{V}_{\mathrm{E}}}{\overline{\mathrm{X}}}
$$

3.11. Dispositif 2.44.3.1 et 2.44.3.2. (1/2 frères, Fig. $\left.1_{A, B}, C\right)$

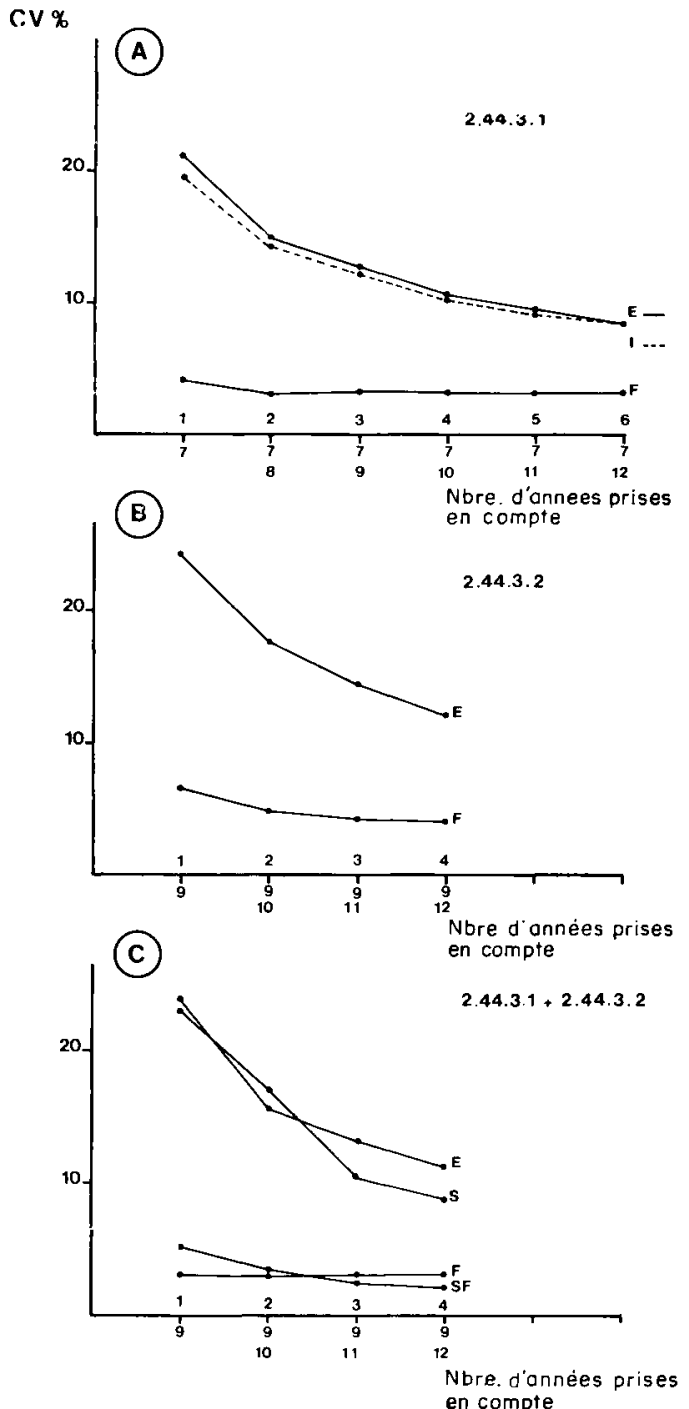

Fig. 1

Evolution des coefficients de variation des composantes de la variance phénotypique en fonction de lordre de cumul des accroissements

En dessous de l'axe des abscisses figurent la première (nombre du haut) et la dernière (nombre du bas) saisons de végétation prises en compte dans le cumul des accroissements.

Evolution of variation coefficients values with the level of sum of height increments.

$V$ alues are given for the different components of the phenotypic variance.

For example, level 2 means sum of two annual successive height increments 
Que ce soit en conditions difficiles (2.44.3.2.) ou en conditions plus favorables (2.44.3.1.) les courbes des coefficients de variation des composantes inter-famille et erreur ont la même évolution :

- chute constante de la composante erreur suivant le cumul des pousses successives. Cette composante erreur comprend à la fois l'effet micro-environnemental à l'intérieur d'une parcelle unitaire et l'effet individu :

- remarquable stabilité de la composante inter malgré une légère décroissance entre le cumul dordre 1 et le cumul d'ordre 2.

En valeur absolue les valeurs de $\mathrm{CV}_{\mathrm{F}}$ et de $\mathrm{CV}_{\mathrm{F}}$ sont légèrement plus importantes dans le 2.44.3.2. En d'autres termes, les différences individuelles et familiales sont plus prononcées en conditions difficiles qu'en conditions favorables.

L'analyse combinée sur les deux dispositifs confirme les précédents résultats et apporte deux informations complémentaires (Fig. 1, ) :

- courbe décroissante de la composante station $\left(\mathrm{CV}_{\mathrm{S}}\right)$;

- courbe décroissante mais de façon moins importante de la composante interaction $\mathrm{F} \times \mathrm{S}\left(\mathrm{CV_{ \textrm {l } }}\right)$

La décroissance du coefficient de variation erreur en fonction de lordre du cumul des pousses pourrait être due à un artefact lié à l'erreur de mesure.

Soit $E_{i, j}=I_{i j}+J_{i j}$

$E_{i j}$ : terme erreur dans les analyses de variance décrites dans le paragraphe 2.

$\mathrm{I}_{\mathrm{i}, \mathrm{j}}$ : effet individu + micro milieu (purgé de l'erreur de mesure $\mathrm{N}\left(0, \mathrm{~V}_{\mathrm{J}}\right)$.

$\mathrm{J}_{\mathrm{ij}}$ : effet erreur de mesure $\mathrm{N}\left(0, \mathrm{~V}_{\mathrm{J}}\right)$ (indépendante de $\mathrm{I}_{\mathrm{ij}}$ ).

Le coefficient de variation du terme erreur s'écrit :

$$
C V_{\mathrm{L}:}=\frac{V V_{\mathrm{E}}}{\mathrm{x}}=\frac{\sqrt{V_{\mathrm{I}}+\bar{V}_{\mathrm{I}}}}{\overline{\mathrm{x}}}
$$

La mesure d'un accroissement quel que soit son ordre de cumul est faite par différence de deux hauteurs. L'erreur absolue de mesure $\mathrm{J}_{\mathrm{ij}}$ est donc la même quel que soit l'ordre du cumul et indépendante de la hauteur. Cette caractéristique pourrait très bien expliquer la décroissance de $\mathrm{CV}_{\mathrm{E}}$ avec l'ordre du cumul, c'est-à-dire avec une augmentation de $\mathrm{X}$.

En supposant que l'écart type de $\mathrm{J}_{\mathrm{ij}}$ soit de $10 \mathrm{~cm}$ (ce qui correspond à la limite supérieure de lerreur de mesure possible) les coefficients de variation de $\mathbf{I}_{\mathbf{j} j}$ ont été reportés sur la figure $1_{\Lambda}$. La courbe de $C V_{I}$ est très proche de $C V_{E}$. L'erreur de mesure n'affecte donc que de manière négligeable $\mathrm{CV}_{\mathrm{E}}$.

Il apparaît donc très nettement que les différences micro-environnementales à l'échelle d'une parcelle unitaire et macro-environnementale entre deux stations (humide et sèche en l'occurrence) jouent un rôle de moins en moins important dans lexpression de la croissance en hauteur dı pin maritime à mesure que celle-ci est appréciéc par un cumul d'accroissements d'ordre plus élevé. D'autre part, en moyenne les différences familiales sont plus prononcées en milieu défavorable qu'en milieu favorable. 

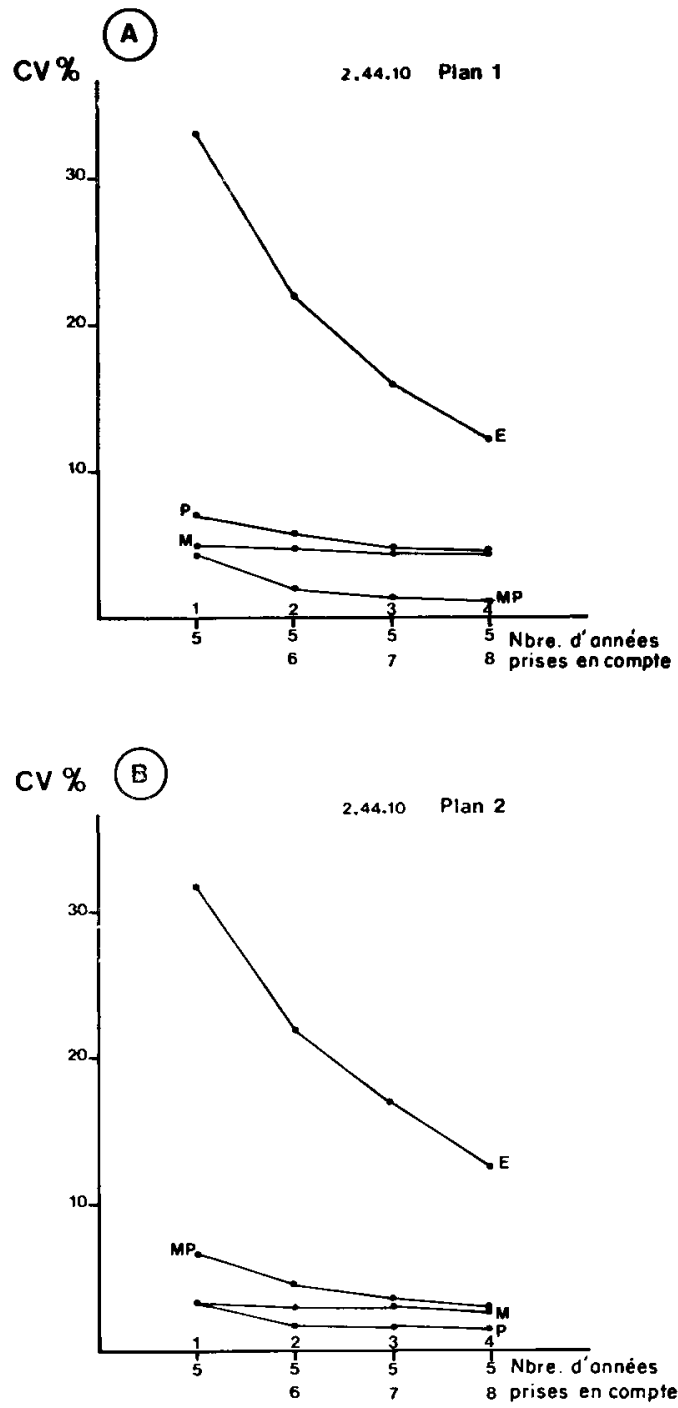

FIG. 2

Evolution des coefficients de variation des composantes de la variance phénotypique en fonction de l'ordre de cumul des accroissements 2.44.10. Plan 1

En dessous de l'axe des abscisses figurent la première (nombre du haut) et la dernière (nombre du bas) saisons de végétation prises en compte dans le cumul des accroissements.

Evolution of variation coefficients values with the level of sum of height increments. Values are given for the different components of the phenotypic variance 


\subsection{Dispositif 2.44 .10 (figure $2_{A, B}$ )}

Compte tenu de la structure parentale (plan de croisement factoriel) la variabilité phénotypique a pu être décomposée en composante mère, père, interaction mère $\times$ père et erreur.

- En ce qui concerne cette dernière, l'évolution de $C_{\mathrm{F}}$ est identique à celle observée dans les dispositifs 2.44.3.1. et 2 .

- La composante mère reste constante et dans les deux plans est de l'ordre de grandeur de celle du père. Ceci indique l'absence d'effets maternels à cet âge (5 à 8 ans).

- La composante père curieusement connaît une décroissance, faible certes, mais constatée dans les deux plans de croisements. La période de mesure correspond dans ce test particulier à la première mise à fleurs du pin maritime. $Y$ aurait-il compétition entre développement des organes végétatifs et organes sexuels, fonctions dans laquelle l'un des génomes (père ou mère) serait plus particulièrement impliqués ?

- La composante interaction $\mathrm{CV}_{\mathrm{MP}}$ décroît régulièrement en fonction des cumuls d'ordre supérieur. Si dans l'un des plans cette composante est faible (plan 1), dans le second plan elle est beaucoup plus élevée. C'est l'information supplémentaire apportée par ces deux plans de croisements. C'est l'une des composantes de la variance phénotypique à laquelle le sélectionneur est le moins intéressé du moins dans le schéma de sélection actuel du pin maritime qui exploite exclusivement la part additive du mode d'action des gènes. Ainsi, en valeur relative, toute source de variation peu utilisée en sélection diminuant les estimations des paramètres génétiques tels que l'héritabilité décroît avec l'ordre du cumul des accroissements.

\subsection{Conséquences sur l'estimation des paramètres génétiques}

\subsection{Dans les deux dispositifs 2.44.3.1. et 2.44.3.2.}

La diminution de la variabilité relative due à la composante erreur $E$ d'une part et la stabilité de la variabilité relative due à la composante familiale $\mathrm{F}$ d'autre part, entraînent une double conséquence, ainsi qu'en témoignent les figures $3_{\mathrm{A}, \mathrm{B}, \mathrm{C}}$ :

- une baisse du coefficient de variation phénotypique;

— et, ipso facto, une augmentation des héritabilités.

Les corollaires de ce résultat sont importants en sélection : en effet dans l'expérimentation, les tests de descendances, le sélectionneur s'attache à maîtriser au maximum la variation environnementale. Cela est possible par la sophistication des dispositifs expérimentaux, mais aussi en ce qui concerne la croissance en hauteur, par le cumul d'un nombre maximum de pousses successives.

\subsection{Dispositif 2.44.10.}

Outre les valeurs d'héritabilité, ce dispositif permet d'estimer les variances dues aux effets d'interaction entre gènes. L'importance de la variance de dominance dans la variance génétique est estimé par le rapport $\mathrm{V}_{\mathrm{D}} / \mathrm{V}_{\mathrm{G}}$. 

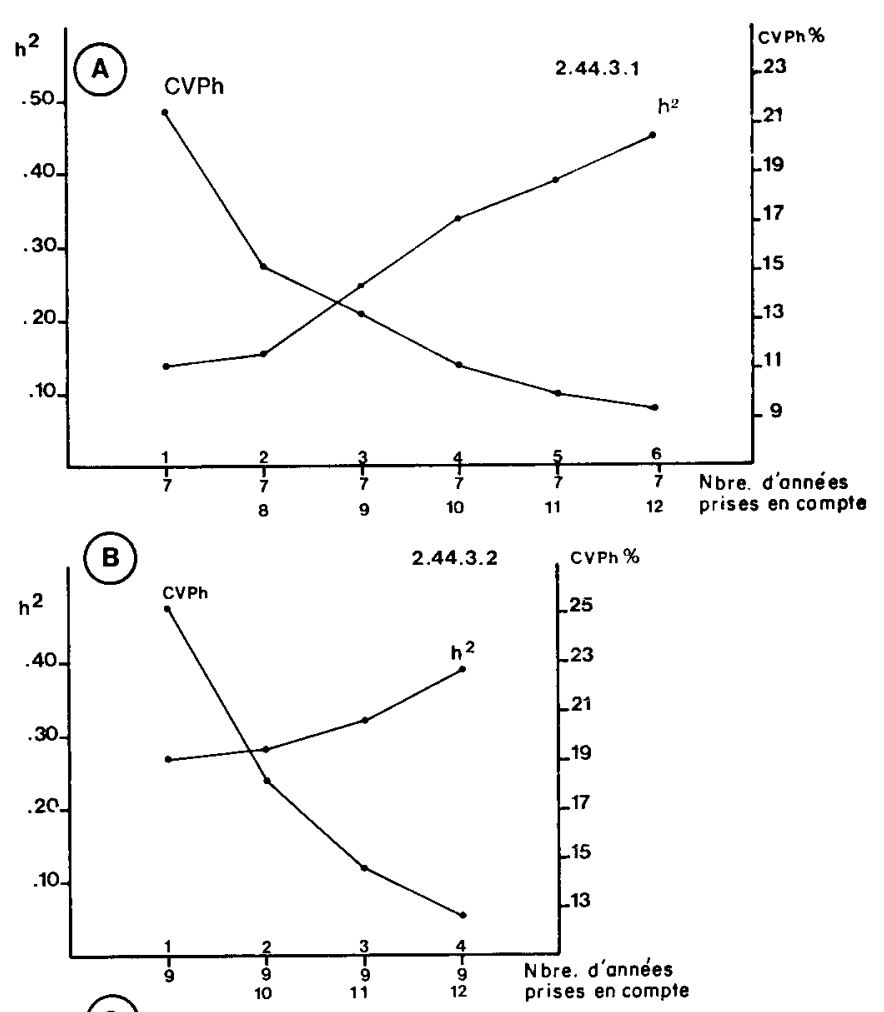

(c)

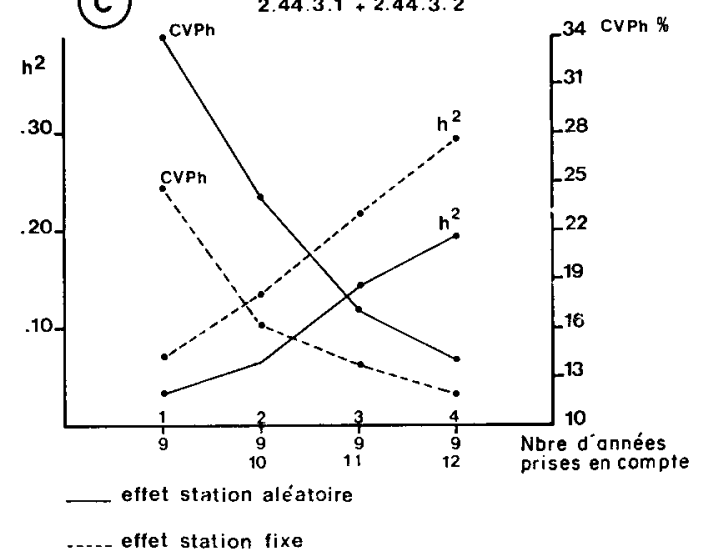

FIG. 3

Evolution du coefficient de variation phénotypique et de l'héritabilité en fonction de l'ordre de cumul

En dessous de l'axe des abscisses figurent la première (nombre du haut) et la dernière (nombre du bas) saisons de végétation prises en compte dans le cumul des accroissements.

Evolution of phenotypic variation coefficient and of heritability with the level of sum of height increments 


\section{- Héritabilités (au sens strict)}

Les valeurs d'héritabilité sont représentées sur les figures $4_{\boldsymbol{A}}$ et $4_{13}$. Chaque courbe joint les points de même ordre de cumul d'accroissements. Par exemple, les points $5,6,7$ et 8 liés par une même courbe correspondent aux accroissements annuels de la $5^{\mathrm{e}}, 6^{\mathrm{e}}, 7^{\circ}$ et $8^{\circ}$ année de végétation (cumul d'ordre 1). En abscisse se trouve la première année du cumul. Le cumul d'ordre 4 est bien évidemment représenté par un seul point.
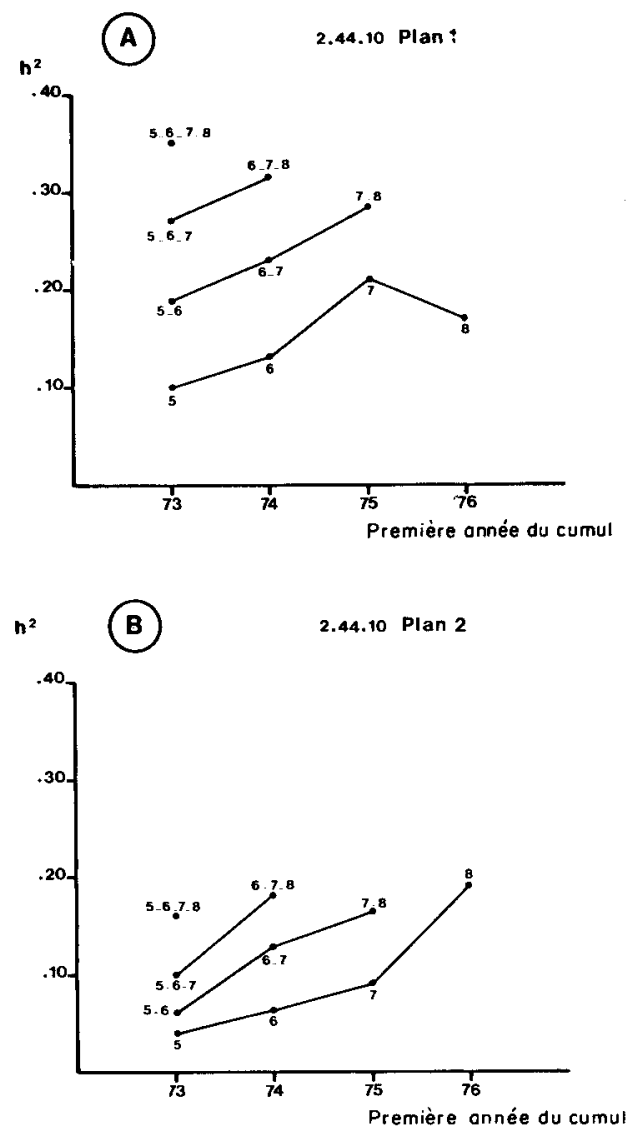

FIG. 4

Evolution des valeurs d'héritabilité en fonction de l'ordre du cumul et de l'âge

En regard de chaque point sont indiquées les années de végétation prises en compte dans le cumul des accroissements. La première correspond à l'année 1973.

Evolution of heritability coefficients with the level of sum height increments and with age

Les résultats issus du test 2.44.3. sont confirmés par ceux du test 2.44.10. à savoir l'augmentation des héritabilités avec l'ordre du cumul d'accroissements. La cause principale de cette évolution paraît être l'âge : tous les cumuls de même ordre subissent une augmentation de l'héritabilité avec l'âge et ceci quel que soit le plan de croisement. 
- Variance de dominance

$$
v_{0} v_{G}^{\%}
$$

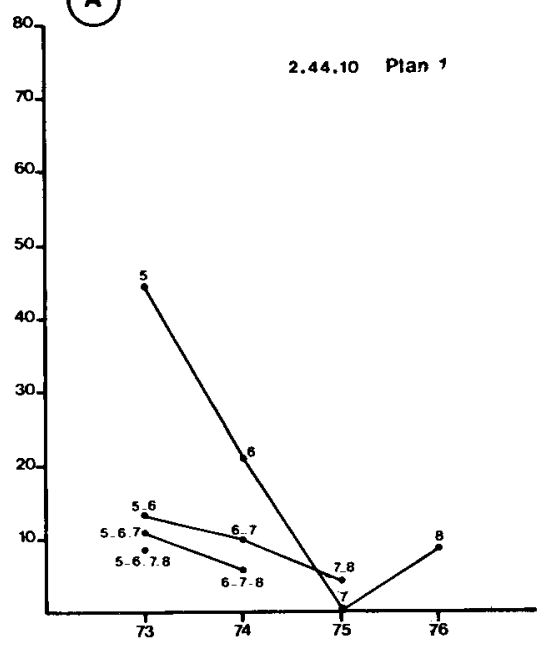

(B)

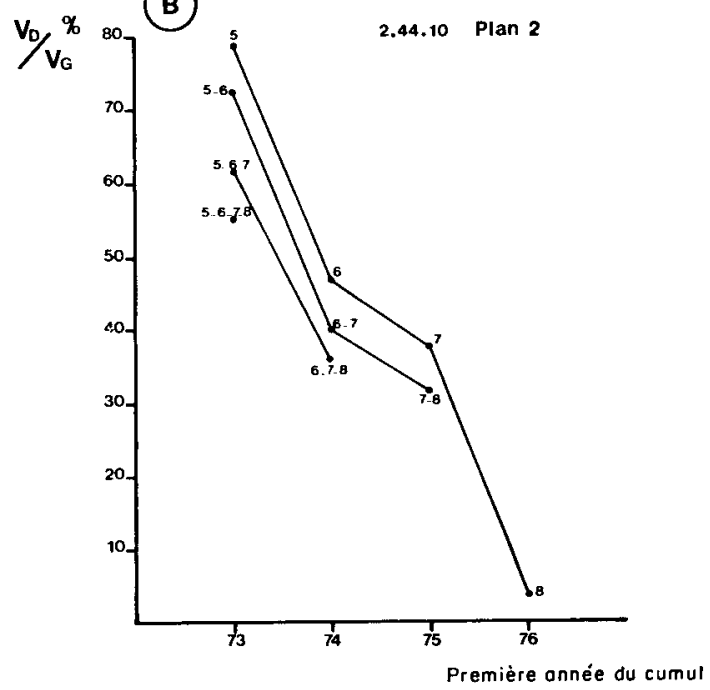

FIG. 5

Evolution du rapport $V_{D} / V_{G}$ en fonction de l'ordre de cumul et de l'âge En regard de chaque point sont indiquées les années de végétation prises en compte dans le cumul des accroissements. La première correspond à l'année 1975.

Evolution of $V_{n} / V_{G}$ values with the level of sum of increments and with age

L'évolution du rapport $\mathrm{V}_{\mathrm{D}} / \mathrm{V}_{\mathrm{G}}$ selon l'ordre des cumuls est représentée sur les figures $5_{\mathrm{A}}$ et $5_{\mathrm{B}}$, respectivement pour le plan de croisement 1 , et le plan de croisement 2. Les lois qui régissent cette évolution sont symétriques de celles correspondant à l'évolution des héritabilités : 
- décroissance avec l'ordre du cumul;

— décroissance avec l'âge.

Ce ne sont là que des corollaires des observations faites dans le paragraphe 3.1.

\subsection{Mode d'action des gènes et aptitude aux combinaisons}

Dans le mode d'action des gènes l'importance relative des effets d'interaction par rapport aux effets additifs est donnée par le rapport $\frac{\mathrm{V}_{1}}{\mathrm{~V}_{\mathrm{A}}}$.

Aux yeux du sélectionneur, la connaissance de la part due à l'aptitude spécifique par rapport à l'aptitude générale de la combinaison $\left(\frac{\mathrm{V}_{\mathrm{A} N \mathrm{C}}}{\mathrm{V}_{\mathrm{A} \text { (xi }}}\right)$ dans la variance phénotypique est un élément de décision dans la stratégie de la sélection.

Dans le cas présent, ces deux rapports sont équivalents. Ce rapport ne correspond pas tout à fait au «degré moyen de dominance» tel qu'il est défini par Comstock \& Robinson (1952) : $\sqrt{\frac{2 V_{b}}{V_{A}}}$. De toute manière, tel qu'il est estimé par ces auteurs, ce rapport n'a de sens que si les fréquences géniques sont de $1 / 2$, hypothèse qui s'ajoute à toutes celles inhérentes au modèle génétique et qui n'est absolument pas vérifiée dans le cas présent. Par ailleurs, la connaissance du "degré moyen de dominance $» n$ 'a aucune signification utile en sélection. Seul le rapport $\frac{V_{A s o}}{V_{A G C}}$ apporte une information utile et correspondant en plus de sa signification statistique à un élément de décision en sélection.

Les valeurs de $\frac{V_{A s}}{V_{A G C}}$ se trouvent dans le tableau 1. Ce tableau tout comme les figures 4 et 5 font apparaître des différences importantes dans le mode d'action des gènes entre le premier et le second plan de croisement. Les paramètres génétiques sont liés au milieu et à la population sur lesquelles ils sont estimés. Dans le cas présent, le milieu est identique puisque les familles des deux plans de croisements se trouvent dans le même dispositif expérimental et ont été installées sans tenir compte de leur appartenance à l'un ou l'autre plan. L'explication peut résider à différents niveaux :

\section{Plan de croisement}

La rectangularité des plans de croisement (4 pères et 21 mères) affecte de la même manière la précision des estimations des variances additives et de dominance (Namkoong, Roberds, 1974). Néanmoins à la suite d'échecs de pollinisation, 3 familles 
ne sont pas représentées dans le plan 1 alors que ce nombre est de 10 dans le plan 2 . Dans le dernier cas la précision de l'estimation de $V_{D}$ est moindre.

\section{Tableau 1}

Estimations de $\frac{V_{A S C}}{V_{A G C}}$ pour 4 cumuls d'accroissements d'années successives Estimation of $\frac{V_{A S C}}{V_{A G C}}$ for successive sums of annual height increments of four successive years

\begin{tabular}{c|c|c|c|c}
\hline \hline Caractère & 73 & $73-74$ & $73-75$ & $\frac{73-76}{}$ \\
\hline Plan $1 \ldots \ldots \ldots \ldots \ldots$ & .77 & .15 & .13 & .10 \\
\hline Plan $2 \ldots \ldots \ldots \ldots \ldots$ & 3.71 & 2.68 & 1.64 & 1.21 \\
\hline
\end{tabular}

Par ailleurs, le faible échantillonnage de pères a pu regrouper dans l'un des plans de croisement des pères aux balances génétiques différentes de ceux du second plan de croisement.

\section{Modèle génétique}

Si les hypothèses de non inbreeding, de non épistasie et d'équilibre de linkage ne sont pas vérifiées, la variance de dominance sera surestimée. L'une ou l'autre de ces hypothèses a très bien pu faillir au plan de croisement 2 .

Si l'aptitude spécifique est importante, la possibilité de la prédire peut guider le choix des croisements à réaliser. L'utilisation d'un modèle généralement employé dans les études d'interaction génotype $\times$ milieu permet de vérifier cette possibilité. Certains auteurs envisagèrent d'ailleurs de l'utiliser pour l'interprétation de plans de croisements factoriels (A.J. WRIGHT, 1971). En se plaçant dans une situation à effets aléatoires : $(M P)_{i j}=D M_{i} P_{j}+B_{i} P_{j}+A_{j} M_{i}+C_{i j}$.

Les estimateurs des $\mathrm{D}, \mathrm{B}_{\mathbf{i}}$ et $\mathrm{A}_{\mathbf{j}}$ sont donnés dans une étude précédente (KREMER, 1980b).

Le programme FLOYD utilisé pour cette interprétation nécessite que toutes les combinaisons entre les deux facteurs principaux soient représentées. Pour ce faire, les parents femelles qui étaient impliqués dans des croisements suivis d'échec de pollinisation ont été supprimés, soient 2 dans le plan de croisement 1 et 6 dans le plan de croisement 2 .

Les sommes des carrés imputables à $D, B_{i}$ et $A_{j}$ sont exprimées en pourcentage de la somme des carrés des écarts du terme interaction dans le tableau 2. Globalement l'aptitude spécifique à la combinaison n'est expliquée qu'imparfaitement par le modèle à en juger par les valeurs du tableau $2(30$ p. 100 à 50 p. 100 de la SCE sont dues à la $\mathrm{SCE}$ de $\mathrm{D}, \mathrm{B}_{\mathrm{i}}$ et $\mathrm{A}_{\mathrm{j}}$ ). Compte tenu de la rectangularité des plans de croisement, la SCE du terme $B_{i}$ est bien évidemment plus importante que celle du terme $A_{j}$. 


\section{Tableau 2}

Pourcentage de la SCE interaction père $\times$ mòre expliqué par le modèle linéaire pour 4 cumuls d'accroissements d'années successives

Percentage of SCE of interaction mother $\times$ father expressed by the linear model for successive sums of annual height increments of four successive years

\begin{tabular}{|c|c|c|c|c|c|}
\hline & & 73 & $73-74$ & $73-75$ & 73-76 \\
\hline \multirow{4}{*}{ Plan 1} & $D \ldots \ldots \ldots$ & $0 \mathrm{NS}$ & $2 \mathrm{NS}$ & $2 \mathrm{NS}$ & $4 \mathrm{NS}$ \\
\hline & $B_{i} \ldots \ldots \ldots$ & $36 \mathrm{NS}$ & $44\left(^{*}\right)$ & $42 \mathrm{NS}$ & $34 \mathrm{NS}$ \\
\hline & $A_{j} \ldots \ldots \ldots$ & $1 \mathrm{NS}$ & $5 \mathrm{NS}$ & $1 \mathrm{NS}$ & $4 \mathrm{NS}$ \\
\hline & $\mathrm{D}+\mathrm{B}_{\mathbf{i}}+\mathbf{A}_{\mathbf{j}}$ & 37 & 5.1 & 45 & 42 \\
\hline \multirow{4}{*}{ Plan 2} & $\mathrm{D} \ldots \ldots \ldots$ & $0 \mathrm{NS}$ & $0 \mathrm{NS}$ & $1 \mathrm{NS}$ & $0 \mathrm{NS}$ \\
\hline & $B_{i} \ldots \ldots \ldots$ & $31 \mathrm{NS}$ & $30 \mathrm{NS}$ & $26 \mathrm{NS}$ & $24 \mathrm{NS}$ \\
\hline & $A_{j} \ldots \ldots \ldots$ & $1 \mathrm{NS}$ & $2 \mathrm{NS}$ & $8 \mathrm{NS}$ & $5 \mathrm{NS}$ \\
\hline & $\mathbf{D}+\mathbf{B}_{\mathrm{i}}+\mathbf{A}_{\mathrm{j}}$ & 32 & 32 & 35 & 29 \\
\hline
\end{tabular}

: test $\mathbf{F}$ significatif au seuil de $10 \%$

Néanmoins, de l'application de ce modèle on peut tirer deux conclusions :

- L'interaction père $\times$ mère (l'aptitude spécifique à la combinaison) ne suit pas un modèle multiplicatif : D n'exprime qu'une partie infime de la SCE interaction. En d'autre termes les parents à plus forte AGC ne sont pas ceux qui «exploitent » au mieux l'AGC de leur partenaire pour donner des familles aux performances très élevées quand l'AGC du partenaire est élevée, mais aussi performances très faibles quand l'AGC du partenaire est faible.

- La partie de l'interaction mère $\times$ père expliquée par le modèle est due essentiellement à $B_{i}$ et à $A_{j}$. Les pentes des droites de régression $\left(B_{i}\right.$ ou $\left.A_{j}\right)$ auraient pu être utilisées pour prédire l'ASC des croisements compte tenu de l'AGC des parents. Cependant, elles ne sont jamais significatives par rapport aux déviations $C_{i j}$. En d'autres termes, les effets réciproques, les interactions noyau-cytoplasme (sousjacentes aux interactions père $X$ mère) ne s'expliquent pas par un modèle linéaire.

A l'appui de ce résultat, on peut remarquer que dans le plan de croisement où la variance de l'aptitude spécifique à la combinaison est la plus importante, le modèle d'A.J. Wright est d'autant moins efficace.

\section{4. - Discussion}

\subsection{Décomposition de la variance phénotypique}

La principale conclusion qui se dégage des résultats du paragraphe 3 est la diminution de limportance relative des composantes de la variance phénotypique 
que le sélectionneur souhaiterait minimiser par ailleurs (erreur, effet station, interaction famille $\times$ station, interaction mère $\times$ père) : voir figures 1 et 2 . Les conséquences au niveau des paramètres génétiques (figures 4 et 5 ) sont alors évidentes : augmentation des héritabilités et diminution du rapport $V_{D} / V_{G}$.

\section{Erreur}

Le terme erreur tel qu'il est estimé dans cette étude comprend à la fois l'effet microstation à l'échelle d'une parcelle unitaire et l'effet individuel propre. Par ailleurs, l'évolution de son coefficient de variation avec l'ordre du cumul peut être le résultat d'un effet âge ou d'un comportement interannuel particulier. Pour vérifier l'importance de l'un ou l'autre de ces facteurs, les $\mathrm{CV}_{\mathrm{E}}$ des accroissements annuels successifs ont été représentés dans la figure 6 pour les trois dispositifs.
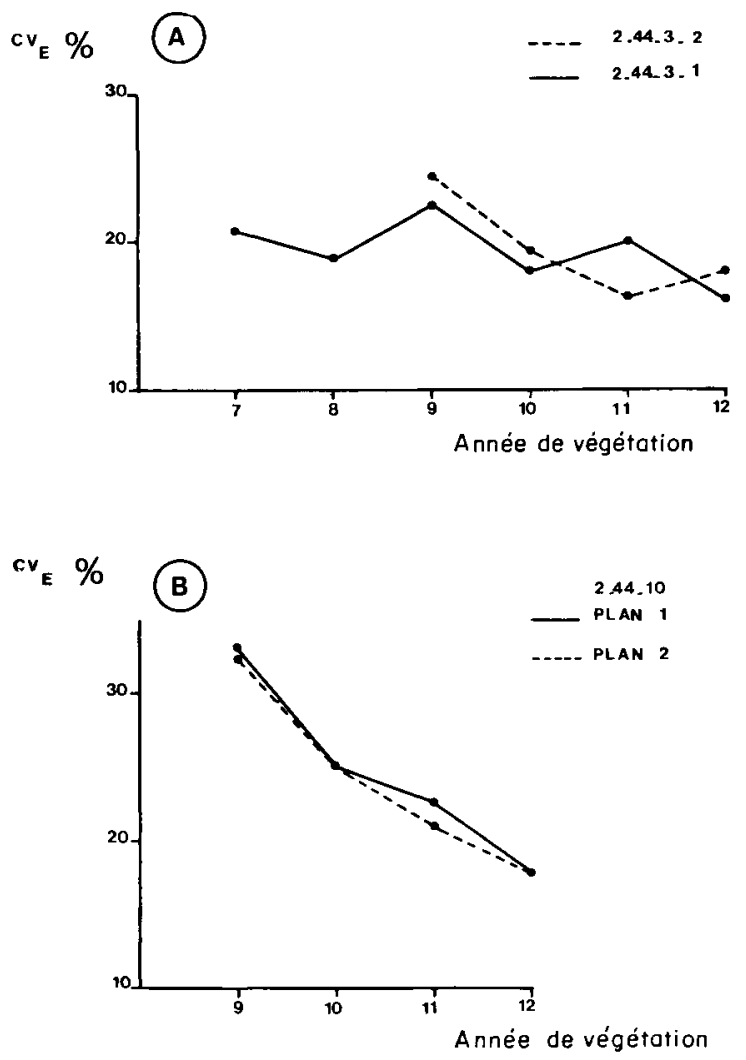

FIG. 6

Evolution du cocfficient de variation du terme erreur en fonction de l'âge

Evolution of error variation coefficient with age

- En ce qui concerne les deux plans de croisements du 2.44.10. (5 $5^{\circ}$ à $8^{\mathrm{e}}$ année de végétation) il semble bien que la diminution $d u C_{\mathrm{E}}$ des cumuls soit due à un effet 
âge, qui correspond ici à la diminution des effets dus à la crise d'installation. Ce résultat entraîne par ailleurs l'augmentation des héritabilités des accroissements annuels successifs (cf. figure 4).

- Après la $7^{\mathrm{e}}$ année de végétation l'importance relative de la composante erreur se stabilise au niveau des accroissements annuels successifs jusqu'à la $12^{\circ}$ année (figure $6_{\Lambda}$ pour les dispositifs 2.44.3.1. et 2.44.3.2.). La diminution de $C_{\mathrm{F}}$ au niveau des cumuls ne peut donc s'expliquer que par un rééquilibrage de la croissance à mesure qu'elle est cumulée sur plusieurs années.

Entre la $5^{\circ}$ et la $8^{\circ}$ année de végétation, l'effet âge peut s'expliquer par les changements de l'environnement externe au niveau d'une parcelle unitaire et de l'évolution interne de l'arbre. En ce qui concerne l'effet microstation, la concurrence herbacée peut induire des différences individuelles considérables de la croissance en hauteur et cela d'autant plus que l'arbre est jeune. Par ailleurs, des attaques de rouille courbeuse peuvent aboutir à des conséquences analogues à cet âge. Enfin, entre 5 et 8 ans le pin maritime abandonne progressivement une croissance de type libre vers une croissance fixe et connaît les premières floraisons à cette époque. Pour autant qu'il existe une variabilité individuelle dans l'expression de chacun de ces processus, les répercussions au niveau de la croissance annuelle peuvent entraîner des différences individuelles importantes.

Entre la $7^{\circ}$ et la $12^{\text {e }}$ année, l'effet âge n'explique pas la diminution du $C_{\mathbf{E}}$ au niveau des cumuls, à en juger par l'évolution des $\mathrm{CV}_{\mathrm{E}}$ des accroissements annuels successifs (figure $6_{\Lambda}$ ). L'utilisation du modèle linéaire d'interaction génotype $\times$ environnement appliquée au niveau individu $\times$ année avait montré qu'il existait une forte variabilité individuelle de la pente de la droite de régression (KREMER, 1981 b). En d'autres termes la variabilité du comportement interannuel au niveau individuel tout en maintenant une importance relative de la composante $\mathbf{E}$ de l'accroissement annuel constante d'année en année, en changeant le classement des individus peut diminuer celle du cumul sur plusieurs années. En fait, tout se passe comme s'il y avait rééquilibrage de la croissance sur plusieurs années. Au plan biologique, les interprétations peuvent être diverses : alternance de la concurrence entre individus au niveau racinaire, production d'un second cycle une année n qui limite l'élongation primaire de l'année $\mathrm{n}+1$ (Kremer, 1981 a).

\section{Effet station et interaction famille $\times$ station}

Une étude précédente (Kremer, 1981 a) montre quil n'y a pas forcément homologie entre années favorables à Saint-Alban (2.44.3.1.) et années favorables à Soustons (2.44.3.2.). En cumulant sur plusieurs années, les différences entre stations deviennent moins importantes ce qui occasionne une diminution de $\mathrm{CV}_{\mathrm{s}}$. L'évolution de la composante famille $\times$ station est elle également liée à cette observation.

\section{Effet interaction mère $\times$ père}

La même référence fait également apparaître que la variance d'interaction mère $X$ père pouvait n'être qu'un artefact lié aux conditions morphogénétiques d'expression de la croissance. En effet en séparant une même année de la population du plan de croisement 1 du 2.44 .10 (à l'intérieur de chaque famille) en souspopulations monocycliques (individus monocycliques) et polycycliques stricts (individus polycycliques) la variance d'interaction $\mathrm{M} \times \mathrm{P}$ était nulle dans chacune des sous- 
populations alors qu'elle ne l'était pas pour la population initiale. Par ailleurs, la mesure du polycyclisme est d'autant meilleure quand elle est faite sur plusieurs années : on tend vers une homogénéisation de la population.

Enfin, et c'est une remarque générale, la pousse annuelle d'une année donnée n'est qu'une expression imparfaite et insuffisante du potentiel de croissance qui ne s'exprime que sur plusieurs années. Au niveau phénotypique, certaines observations (Kremer, 1980a) montraient que le second cycle de l'élongation d'une année $\mathbf{n}$ donnée pouvait influer sur le premier cycle de l'élongation de l'année suivante $\mathbf{n}+1$. Cette étude vient de montrer que les facteurs limitant l'expression de la croissance une année donnée sont essentiellement des facteurs du milieu.

L'effet parental (mère ou père) reste toujours constant au moins pendant la période au cours de laquelle les accroissements ont été mesurés. Au niveau de la sélection, ces remarques sont résumées de la manière suivante : l'accroissement d'un cumul d'ordre $n$ est un meilleur prédicteur d'un accroissement de cumul d'ordre inférieur que ce dernier accroissement lui-même. Ceci est illustré par la matrice des coefficients de prédiction génétique des six différents cumuls d'accroissements successifs dans le 2.44.3.1. (tableau 3). Le CGP intervient directement dans l'expression du gain génétique espéré (BARADAT, 1976).

\section{TABleau 3}

Matrice des coefficients de prédiction génétique pour 6 cumuls d'accroissements d'années successives 2.44.3.1.

Coefficients of genetic prediction Matrix of differents sums of annual height increments of six successive years

\begin{tabular}{|c|c|c|c|c|c|c|}
\hline & 71 & $71-72$ & $71-73$ & $71-74$ & $71-75$ & $71-76$ \\
\hline 71 & .14 & & & & & \\
\hline $71-72$ & .14 & .15 & & & & \\
\hline $71-73$ & .15 & .18 & .25 & & & \\
\hline $71-74 \ldots \ldots$ & .17 & .20 & .28 & .34 & & \\
\hline $71-75 \ldots \ldots$ & .16 & .20 & .29 & .35 & .39 & \\
\hline $71-76 \ldots$ & .18 & .22 & .31 & .38 & .41 & .45 \\
\hline
\end{tabular}

Dans le cas de la sélection massale, le gain génétique relatif espéré sur le caractère $\mathrm{k}$ quand la sélection porte sur le caractère 1 est :

$$
\begin{aligned}
& 1 \Delta \mathrm{G}_{\mathrm{k}} / \mathrm{G}_{\mathrm{k}}=\mathrm{i}_{1} \mathrm{CGP}_{1, \mathrm{k}} \sigma \mathrm{Ph}_{\mathrm{k}} \\
& \mathrm{CGP}_{\mathrm{l}, \mathrm{k}}=\frac{\operatorname{Cov}\left(\mathrm{A}_{1}, \mathrm{~A}_{\mathrm{k}}\right)}{\sigma \mathrm{Ph}_{1} \times \sigma_{\mathrm{Ph}_{\mathrm{k}}}}
\end{aligned}
$$


D'où l'efficacité de la sélection indirecte par rapport à la sélection directe $\frac{\mathrm{l} \Delta \mathrm{G}_{\mathrm{k}}}{\mathrm{k} \Delta \mathrm{G}_{\mathrm{k}}}=\frac{\mathrm{CGP}_{\mathrm{l}, \mathrm{k}}}{\mathrm{CGP}_{\mathrm{k}, \mathrm{k}}}$ en faisant 1'hypothèse que $\mathrm{i}_{\mathrm{lk}}=\mathrm{i}_{\mathrm{l}}$.

$\mathrm{i}_{\mathrm{k}}$ : intensité de sélection de $\mathrm{k}$.

$\operatorname{cov}\left(A_{1}, A_{k}\right)$ : covariance entre valeurs additives de 1 et de $k$.

$\sigma_{P_{h}}$ : écart type phénotypique du caractère $\mathrm{k}$.

Dans chaque colonne $k$ tableau 3 , pour $1>k$ les valeurs de $\frac{\mathrm{CGP}_{1 . k}}{\mathrm{CGP}_{\mathrm{k}, k}}$ sont supérieures à 1 .

Le meilleur pouvoir prédicteur de 1 vient essentiellement du maintien de très bonnes corrélations entre 1 et $\mathrm{k}$ et d'une héritabilité plus élevée de 1 .

Il n'existe dans la littérature que très peu d'exemples sur l'évolution des composantes de la variance phénotypique. Seuls Namkoong \& Conckle (1975) et Namkoong, USANIS \& SILEN (1972) ont publié des résultats respectivement sur Pinus ponderosa et Pseudotsuga Menziesii. Dans le premier cas, bien que la période sur laquelle a porté l'étude soit plus longue, les résultats obtenus sont proches de ceux exposés dans la présente étude à savoir :

- diminution de l'importance relative du terme erreur;

- maintien de l'importance relative de la variance génotypique jusqu'à 7 ans, chute brutale et nouveau plateau de 8 à 29 ans, terme de l'étude. Cette dernière évolution n'est pas pour autant expliquée.

Le second cas prolonge en quelque sorte notre étude dans la mesure où elle concerne une période plus longue. Le résultat le plus intéressant est le maintien d'une variance génotypique importante jusqu'au stade où les arbres ont surmonté la pression du milieu (vers 15 ans), période qui se situe bien avant que la courbe de la croissance n'atteigne l'asymptote. En tout état de cause, la présente étude mériterait d'être poursuivie sur du matériel plus âgé.

\subsection{Décomposition de la variance génotypique}

Le second résultat intéressant est l'importance des effets additifs des gènes dans le déterminisme de la croissance en hauteur, même si les résultats divergent entre les deux plans de croisement.

En valeur absolue, si l'on supprime la pousse de l'année 1973 , les valeurs de $\mathrm{V}_{\mathrm{D}} / \mathrm{V}_{\mathrm{G}}$ ne sont jamais très élevées (entre 0 et 20 p. 100 dans le plan 1 et 3 et 45 p. 100 dans le plan 2 : voir figure 5). Ces valeurs sont proches de celles trouvées dans d'autres plans de croisements pour la croissance en hauteur (KREMER, 1976). Là encore, les résultats obtenus sur d'autres pins sont peu nombreux. A 4 ans, sur Pinus Virginiana le rapport $V_{\mathrm{D}} / \mathrm{V}_{\mathrm{G}}$ est de $38 \mathrm{p} .100$ pour la hauteur totale (A.G. CorriveaU, 1974). De même sur Pinus taeda la variance de dominance représente une part nettement moins importante de la variance génétique à l'âge de 8 ans $\left(V_{D} / V_{G}\right.$ varie de 0 à 35 p. 100) (D.I. Matziris, 1974). Par contre, chez ces mêmes auteurs dans certains tests de descendances, plus rares certes, ces valeurs sont plus élevées : 80 p. 100 sur Pinus 
taeda à 4 ans (A.G. Corriveau, 1974, P.I. Matziris, 1974). Cette dispersion des valeurs n'est pas surprenante : les paramètres génétiques sont liés à la population et au milieu sur lesquels ils sont estimés. Par ailleurs, cette étude a montré que leurs estimations peuvent varier au cours du temps. En particulier, que ce soit dans le plan de croisement 1 ou dans le plan de croisement 2 la prise en compte de certaines années dans le cumul peut faire varier considérablement l'estimation de VD (année 1973). Néanmoins, en ce qui concerne le pin maritime, le nombre de résultats issus de différents plans de croisement permet de justifier la stratégie de sélection actuellement utilisée qui n'exploite que la partie additive des gènes.

\section{Conclusion}

La croissance juvénile du pin maritime est caractérisée par une variabilité du terme erreur importante, qui ne permet pas de sélectionner efficacement à ce stade. Cette variation (en valeur relative) décroît avec l'âge jusqu'à 8 ans, la variabilité génotypique restant constante. L'héritabilité de la croissance en hauteur augmente à mesure que les accroissements sont cumulés sur un nombre important d'années. La situation actuelle qui consiste à sélectionner en prenant pour critère prédicteur le cumul d'accroissement au-delà de 5 ans paraît donc être un bon compromis entre l'efficacité de la sélection et le développement ultérieur du programme d'amélioration qui demande un raccourcissement des délais d'appréciation des génotypes. Néanmoins, les informations issues d'autres espèces forestières et recouvrant des périodes plus longues demandent à suivre l'évolution des composantes de la variance phénotypique. $\mathrm{Si}$ la variance $\mathrm{AGC}$ représente la plus grande part de la variance génotypique dans la croissance en hauteur du pin maritime, la part de la variance ASC pourrait encore être diminuée par la possibilité certes faible de prédire l'aptitude spécifique à la combinaison de deux parents à partir de leur aptitude générale.

Reçu pour publication en août 1980.

\section{Remerciements}

Les programmes ENVIR, PINEDE, CASOAR font partie de la programmathèque du laboratoire d'amélioration des arbres forestiers. Ils ont été écrits par Ph. Baradat. Je le remercie pour sa disponibilité et son aide qu'il m'a manifestées lors de l'utilisation de ces programmes.

\section{Summary \\ Genetic control of height growth in Pinus pinaster \\ (Pinus pinaster Ait)}

III. - Evolution of the components of the phenotypic and genotypic variance

Componenis of the phenotypic and the genotypic variances of height growth are followed by using the coefficient of variation expressed at each level. Height growth is measured by successive increments between 5 and 12 years. Residual variance, that means error variance, 
environmental variance family $x$ environmental variance and mother $\times$ father interaction decrease with age while main effect variances remain stable. Some of this results are explained by changes in environmental conditions with time. The heritability estimites increase with age while the ratio $V_{1} / V_{a}$ decreases. This is true in so far that height increments of $n$ successive years is more predictive of height increments of $m$ successive years $(m<n)$ than this latest trait itself. Height growth is affected more by additive genes clfects than by non additive genes effects. Possibility to predict specific combining ability (ASC) by using general combining ability $\left(A C C^{C}\right)$ of both parents is discussed by using a gerreal model used in genotype - environment problems.

\section{Key-words}

Genetic model, phenotypic, genotypic, additive dominance variance, general combining ability, specific combining ability, heritability, coefficient of genetic prodiction

\section{Références bibliographiques}

Baradat Ph., 1976. Use of Juvenile - mature relationships and informations from relative in combined multitrait selection. I.U.F.R.O. Advanced generation breding, Bordeaux, 14-18 juin 1976, pp. 121-138.

Corriveau A.G., 1974. The clonal performance of Loblolly and Virginiana Pines, a reflection of their breeding value. Ph. Dissertation, North Carolina State University, Raleigh N.C.

Comstock R.E., Robinson H.F., 1952. Estimation of average dominance of genes. Heterosis, Iowa State College Press, Ames, Iowa.

Hinkelman K., Stern K., 1960. Kreuzungspläne zur Selekti-onszüchtung bei Waldbäumen. Silvae Genet., 9, 121-133.

Kremir A., 1976. Contrôle génétique de la croissance en hauteur chez le pin maritime. Rôle des interactions entre gènes et de l'interaction génotype $\times$ environnement. Mémoire de fin d'études à l'E.N.I.T.E.F., juin 1976.

Kremer A., 1981a. Déterminisme génétique de la croissance en hauteur du pin maritime. I. Rôle du Polycyclismc. Ann. Sci. for., 38 (2), 199-222.

Kremis A., 1981b. Déterminisme génétique de la croissance en hauteur du pin maritime. II. Comportement interannuel. Interaction génotype $X$ année. Alln. Sci. for., 38 (3), $331-355$.

Matziris D.I., 1974. Predicted versus realized Gain in Loblolly Pine (Pinlss taeda L) improve. ment. Ph. D. Dissertation, North Carolina State University, Raleigh N.C.

Namkoong G., Usanis A., Silen R.R., 1972. Age-related variation in genetic control of height growth in Douglas-fir. Theor. appl. Genet., 42, 151-159.

Namkoong G., Roberds J.H., 1974. Choosing mating designs to efficiently estimate genetic variance components for trees. Silvae Genet., 23, 1-3, 43-53.

Namkoong G., Conkle M.T., 1976. Time trends in genetic control of height growth in ponderosa pine. For. Sci., 22, 2-13.

Wrighr A.J., 1971. The analysis and prediction of some two factor interactions in grass breeding. J. agric. Sci., 76, 301-306. 